\title{
Processing Technology and Resveratrol Rich Extract from By-products in Wine Industry
}

\author{
ANCUTA TRIFOI ${ }^{1,2}$, TIMEA GHERMAN ${ }^{1,2}$, OLIMPIU BLAJAN ${ }^{1,2}$, MYRAT VELNAZAROV ${ }^{1}$, RAMI DOUKEH ${ }^{3 *}$ \\ ${ }^{1}$ Research Institute For Auxiliary Organics Products SA, 8 Carpati Str., 551022, Mediaș, Romania \\ ${ }^{2}$ Salmed Farma SRL, 8 Carpat Str.i, 551022, Mediaș, Romania \\ ${ }^{3}$ National Institute for Research Development for Chemistry and Petrochemistry- ICECHIM-Bucuresti, 202 Splaiul \\ Independettei, 060021, Bucharest, Romania
}

\begin{abstract}
Wine production is accompanied with the generation of tremendous amounts of valuable by-products rich in phenolic bioactive compounds. The valorization of these by-products represents a key point in winery, not only because of the health related benefits but also for environmental, by means of reuse of wastes. This paper describes a study on grape marc processing, the by-product of the winery and its valorization to a high added value product: resveratrol concentrated extract. The most efficient method for extracting resveratrol from grape skin comprises the following steps: maceration of the raw material in alcohol, reflux extraction, acid hydrolysis, liquid liquid extraction and resveratrol rich extract recovery. The extract content in resveratrol was determined by HPLC with a UV-Vis detector.
\end{abstract}

Keywords: resveratrol, grape marc, winery by-products

Wine production is currently one of the most important agricultural activities in the world. Romanian wine production in 2015 is placed on sixth position at European level after Italy, Spain, France, Germany, and Portugal and on twelfth position in the world $[1,2]$. Wine production is accompanied with the generation of tremendous amounts of valuable by-products rich in phenolic bioactive compounds. The estimated amount of wastes (steams, seeds, peels and marcs) is $1 \mathrm{~kg}$ of grape pomace for each $6 \mathrm{~L}$ of wine, which is mainly destined to animal feed and for compost elaboration, so the valorization of these by-products represents a key point in winery, not only because of the health related benefits of the compounds but also for environmental, by means of reuse of wastes [2,3]. Resveratrol is a strong polyphenolic antioxidant, considered to be 4-5 times stronger than beta carotene, 50 times stronger than Vitamin E and 20 times stronger than Vitamin $\mathrm{C}$ which is found in grape and wine and in other families of plants such as peanuts, tree bark, Polygonum cuspidatum, a well-known traditional Chinese medicine [4]. Various methods for resveratrol extraction have been tested. These methods include, but are not limited to, conventional solvent extraction [5-7], microwave accelerated extraction [8], ultrasound extraction [9,10], supercritical fluid extraction [11], microbial extraction [12] and even a combination of the those. The main disadvantage of these processes is that beside resveratrol, other components are extracted from the plants and the majority of the studies present only the extraction of the resveratrol and not the isolation too. The most commonly used method for resveratrol isolation is chromatographic column purification. This method is based on the adsorption, distribution and different affinity of the components in a mixture. The stationary phases used are silica gel and polyamide gel. Silica gel columns have low cost and high separation capabilities, but operation is complex, with low yields, requiring recrystallization and subsequent purification. Macroporous resins have a high adsorption selectivity, can be reused, mild desorption conditions and low costs. Resins used for resveratrol purification are NKA-9 [13], H1020 [14], AB-8 [15] and HP500 [16].

This paper presents a grape marc (the by-product of the winery) processing technology and its valorization to resveratrol concentrated extract, by simultaneous solvent extraction and resveratrol purification by bleaching the extracts using TONSIL bleaching earth and recovery of resveratrol by insolubilisation in water.

\section{Experimental part}

Reagents and materials

The solvents used for the extraction were $95 \%$ ethanol, methanol, ethylacetate, methyl tertbutylether. Acetonitrile was of HPLC grade. Water was a reverse osmosis purified water. The reference compounds of trans-resveratrol and polydatin were of purity higher than $99,5 \%$. Grape marc sample - belonging to Vitis vinifera L., was achieved after processing red grapes

*email:ramidoukeh@yahoo.com 
from Târnava, Sibiu County, Romania. The grape marc was processed for separating the grape peel, from the grape seed, dried and milled.

\section{Resveratrol extraction}

Resveratrol extraction from grape peel, was made starting with a method proposed by Wang et al. [7], who studied the extraction of resveratrol form Polygonum cuspidatum a well-known traditional Chinese medicine and comprises the following steps: maceration of the raw material in alcohol, reflux extraction, acid hydrolysis, liquid-liquid extraction, color removal . and resveratrol precipitation.

$100 \mathrm{~g}$ of dried and grounded peel has been dispersed in alcohol (methanol or ethanol), in $1000 \mathrm{ml}$ alcohol and the mixture was left to macerate at room temperature for 12-36 h. After sitting at room temperature, the whole mass was extracted at about $80^{\circ} \mathrm{C}$, for $1-2 \mathrm{~h}$, under continuously stirring, at $400 \mathrm{rpm}$. After reflux extraction, the mixture was filtered and the extract solution was concentrated under vacuum. In order to hydrolyze polydatin to resveratrol, the concentrated extract was dispersed in water, in an extract:water ratio of 1:10. The aqueous solution was adjusted with hydrochloric acid to $p \mathrm{H} 1$ and hydrolyzed for $20 \mathrm{~h}$, at $60^{\circ} \mathrm{C}$. To recover the resveratrol from hydrolyzed aqueous solution, liquid-liquid extraction was used. The aqueous solution was mixed in a separation funnel with an organic solvent (methyltertbutyleter or ethyl acetate), in a 1/1 volumetric ratio. For a high recovery, extraction was repeated three times. For an advanced purification, the extracts were bleached with TONSIL bleaching earth and then resveratrol was recovered by insolubilisation in water and filtered out. The contents of resveratrol and polydatin were detected by HPLC-UV Vis and expressed as $\mathrm{mg} / \mathrm{l}$ of solvent.

\section{Analytical procedure}

Resveratrol and polydatin were separated in a C18 Phenomenex Nucleosil 100 column ( $25 \mathrm{~cm} \times 3,2 \mathrm{~cm}, 5 \mu \mathrm{m})$, using an HPLC system ( Jasco -980) equipped with an UV-Vis detector. The mobile phase consisted of purified water : acetonitril $=50: 50$, in an isocratic mode. The flow rate was $0.7 \mathrm{~mL} / \mathrm{min}$ and the injection volume was $5 \mu 1$. Detection was performed at 300 $\mathrm{nm}$. The concentration of resveratrol and polydatin were quantified at the base of the peak areas and calibration curves were derived from the commercially available standard of trans-resveratrol and polydatin (Sigma). A Metertech UV 208 spectrophotometer has been used to measure the absorbance of the bleached solution, at two wavelengths of $500 \mathrm{~nm}$ and $650 \mathrm{~nm}$.

\section{Results and discussions}

\section{Resveratrol extraction}

Extraction of resveratrol from grape skin was performed using methanol or ethanol, by maceration for one to three days and by reflux extraction. Table 1 comprises the extraction experiments with detailed parameters. The resveratrol concentration increased in the first 2 days of maceration with methanol and then began to decrease slightly. In case of maceration with ethanol, the resveratrol concentration increased by $9.3 \mathrm{mg} / \mathrm{l}$ in the $3^{\text {rd }}$ day compared to $2^{\text {nd }}$ day (Figure 1 ). Resveratrol concentration in methanol extract after 2 days of maceration was $31.7 \mathrm{mg} / \mathrm{l}$ (probe 5) and in ethanol extract after 3 days, it was only $4.7 \mathrm{mg} / \mathrm{l}$ (probe 3 ). When reflux extraction was proceeded, after $1 \mathrm{~h}$, the resveratrol concentrations in the two solvents were very close, $72.4 \mathrm{mg} / \mathrm{l}$ (in methanol) and $71.13 \mathrm{mg} / \mathrm{l}$ (in ethanol). After 2 hours of reflux extraction the concentration in resveratrol increased insignificantly.

Table 1

EXTRACTION EXPERIMENTS

\begin{tabular}{|l|c|}
\hline crt. & Description \\
\hline 1 & Maceration, 1 day; ethanol, room temperature \\
\hline 2 & Maceration, 2 days; ethanol, room temperature \\
\hline 3 & Maceration, 3 days; ethanol, room temperature \\
\hline 4 & Maceration, 1 day; methanol, room temperature \\
\hline 5 & Maceration, 2 days; methanol, room temperature \\
\hline 6 & Maceration, 3 days; methanol, room temperature \\
\hline 7 & Reflux extraction, methanol -1 h \\
\hline 8 & Reflux extraction, ethanol $-1 \mathrm{~h}$ \\
\hline 9 & Reflux extraction, ethanol $-2 \mathrm{~h}$ \\
\hline
\end{tabular}




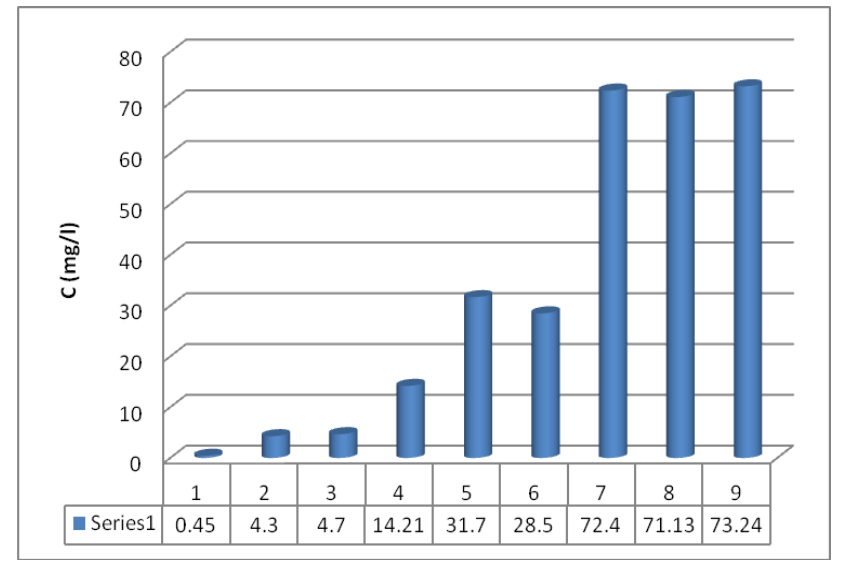

Fig. 1. Comparation between resveratrol concentration in alcoholic extracts. Dried grape skin/alcohol

In order to improve the recovery of resveratrol, the polydatin has been hydrolized to resveratrol by acid hydrolysis $(p \mathrm{H}=1)$, for $20 \mathrm{~h}$. Figure 2 shows the chromatograms of the hydrolysed extracts after $8 \mathrm{~h}$ and after $20 \mathrm{~h}$ of hydrolysis. It can be observed how polydatin peak deacreses with time, while resveratrol peak increases, showing that most polydatin was transformed to resveratrol.

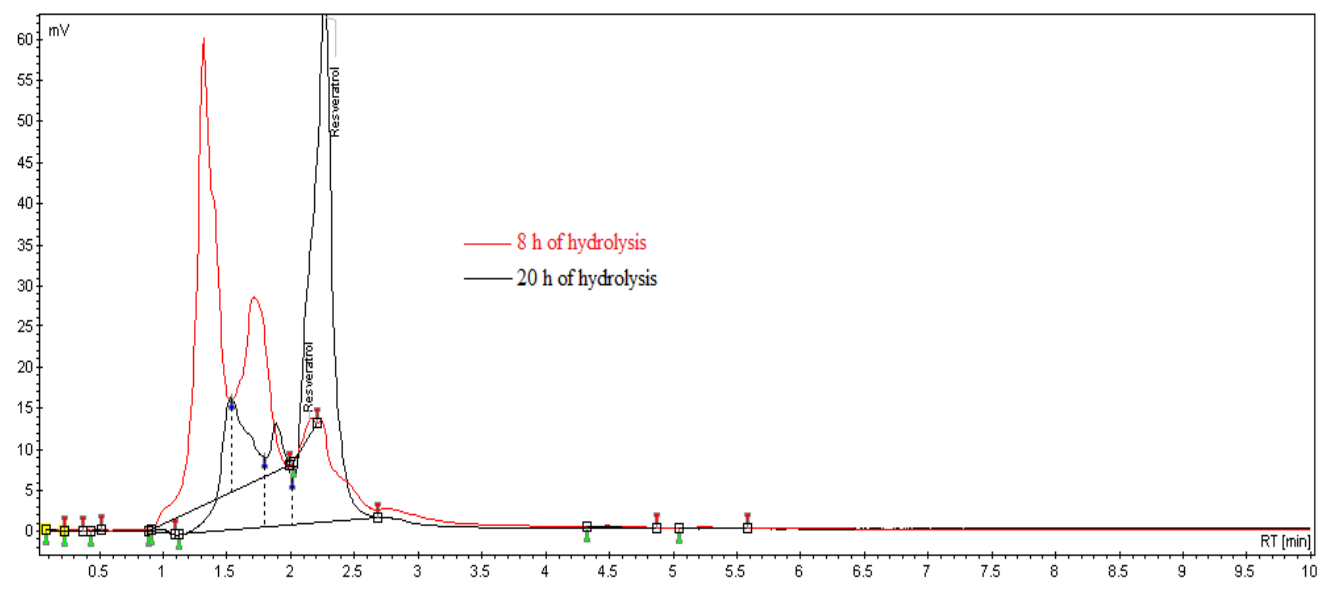

Fig. 2. HPLC chromatograms of samples in aqueous hydrolysed extract

Methyl tert-butyl ether and ethylacetate were tested in the extraction of resveratrol from aqueous hydrolysed extract, in a $1 / 1$ solvent/extract volume ratio. The quantities of recovered resveratrol were $16.98 \mathrm{mg} / \mathrm{l}$ in methyl tert-butyl ether and $41.54 \mathrm{mg} / \mathrm{l}$ in ethylacetate, showing that resveratrol was selectively extracted into the organic phase, with a purity of $82 \%$ and the water soluble impurities mostly remained in the aqueous phase. For ethylacetate, resveratrol recovery was 2.44 times higher than for methyl tert-butyl ether.

For an advanced purification, the extracts were bleached with TONSIL bleaching earth. Process efficiency was monitored by measuring the absorbance of the solution by UV-Vis spectrometry at two wavelengths of $500 \mathrm{~nm}$ and 650 $\mathrm{nm}$, and by analysing the resveratrol concentration. The studies were conducted on a final extract of resveratrol in ethyl acetate. The concentration of the starting solution in resveratrol was $14.07 \mathrm{mg} / \mathrm{l}$ resveratrol and the absorbance of the solution was 0.4651 at $650 \mathrm{~nm}$ and 1.33 at $500 \mathrm{~nm}$.

To determine the influence of the contact time of the solution with the bleaching earth, the process was procedeed for 90 minutes at room temperature using $0.5 \mathrm{~g}$ TONSIL to $100 \mathrm{ml}$ solution. According to Figure 3 the absorbance of the initial solution at $650 \mathrm{~nm}$ dropped from 0.4651 to 0.2448 after 20 minutes and at 0.2207 after 40 minutes. After 40 minutes the absorbance varied insignificantly. This it was also observed at $500 \mathrm{~nm}$ when the absorbance decreases from 1.33 initially to 0.453 after 20 minutes and at 0.381 after 40 minutes of contact. In contrast, by analyzing the resveratrol concentration by HPLC, its concentration decreased very little $(2.2 \%)$, from $14.07 \mathrm{mg} / \mathrm{l}$ to $13.75 \mathrm{mg} / \mathrm{l}$, indicating that resveratrol is not retained on TONSIL and remains in the extract which is further processed. 


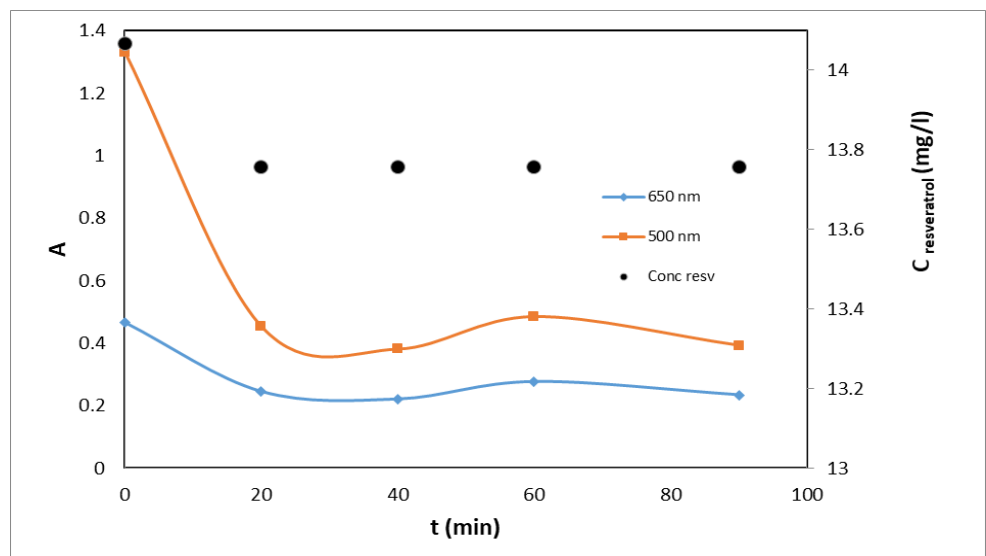

Fig. 3. The influence of contact time with TONSIL bleaching earth on absorbance and resveratrol concentration of resveratrol extracts. $0.5 \mathrm{~g}$ tonsil $/ 100 \mathrm{ml}$ solution, room temperature

The amount of TONSIL influenced the degree of bleaching of the resveratrol extract. Absorbance of resveratrol extract decreased significantly for an amount of TONSIL $>0.3 \mathrm{~g} / 100 \mathrm{ml}$ solution and at $0.5 \mathrm{~g} / 100 \mathrm{ml}$ the absorbance decreased more than $50 \%$, while the resveratrol concentration was constant. At higher amounts of TONSIL of $1 \mathrm{~g} / 100 \mathrm{ml}$, the absorbance of the solution reached 0.0836 (at $650 \mathrm{~nm}$ ) and 0.085 (at $500 \mathrm{~nm}$ ). Using quantities higher than $3 \mathrm{~g}$ of TONSIL/100 ml solution, the absorbance didn't deacrease anymore, indicating that a grater amount of bleaching earth does not affect the color of the solution. At $3 \mathrm{~g} / 100 \mathrm{ml}$ the absorbance of the solution decreased by $87.18 \%$ at $650 \mathrm{~nm}$ and by $95.73 \%$ at 500 $\mathrm{nm}$, after 40 minutes of contact time (figure 4).

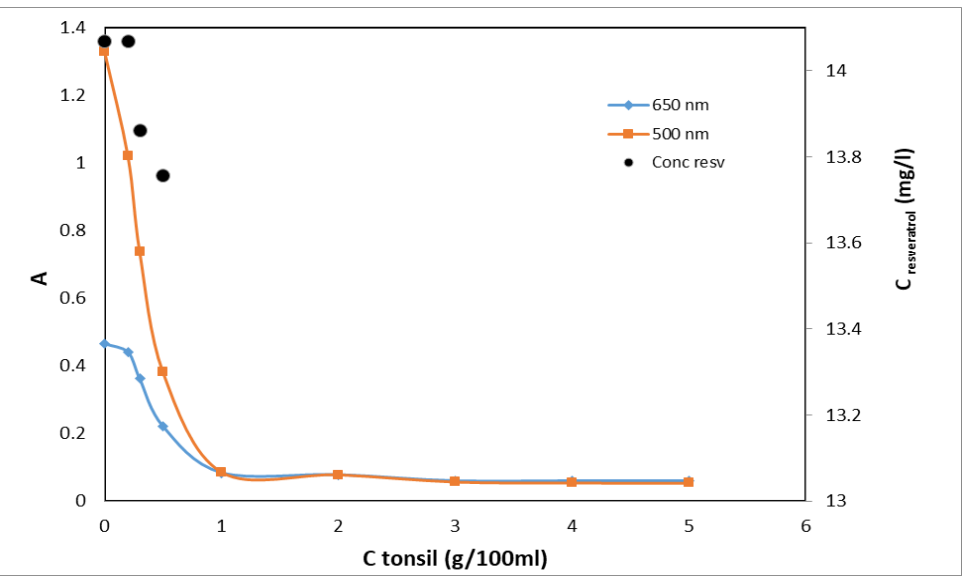

Fig. 4. The influence of TONSIL quantity added to the solvent extract

Temperature had no influence on the bleaching process of resveratrol extracts, using TONSIL. For $3 \mathrm{~g}$ TONSIL/100 ml solution, with an absorbance of 0.4651 at $650 \mathrm{~nm}$ and 1.33 at $500 \mathrm{~nm}$ and a contact time of $40 \mathrm{~min}$, an increase in the temperature from $25^{\circ} \mathrm{C}$ to $40{ }^{\circ} \mathrm{C}$ did not deacrease the absorbance of the solution. It can be observed from figure 5 that there is no difference of the absorbances at $25^{\circ} \mathrm{C}$ and at $40^{\circ} \mathrm{C}$. The purity of the resveratrol in the extract was $92 \%$. 


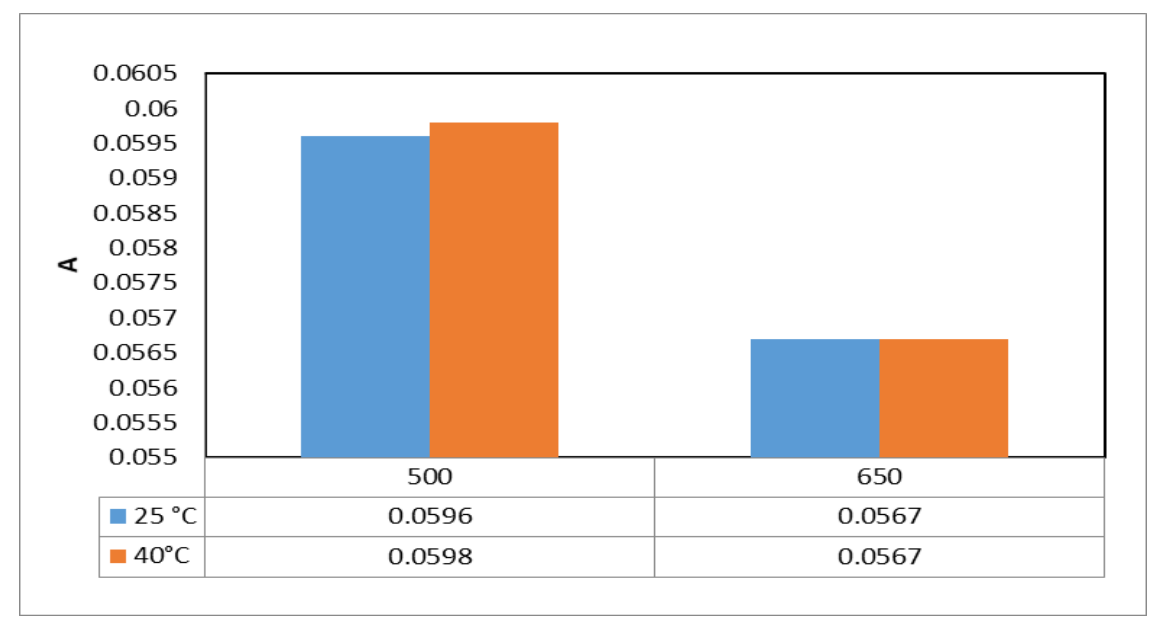

Fig. 5. The influcence of temperature on bleaching process of resveratrol extracts

TONSIL bleaching earth was filtered out and the solvent was 95\% removed by vacuum distillation. Distilled water was added in an extract/water ratio of up to 0.02 , to insolubilise the resveratrol in water. The resulted yellowish crystals have been isolated by filtration.

\section{Conclusions}

Extraction of resveratrol from grape skin has been made using a simple succeeding alcohol extraction, polydatin hydrolysis, solvent extraction, extract bleaching and resveratrol recovery by insolubilisation in water. The recovery of resveratrol in the alcohol extraction phase was best performed after $1 \mathrm{~h}$ of reflux extraction, resveratrol concentrations in the methanol and ethanol were very close. The resveratrol recovery was improved by acid hydrolysis of polydatin, most polydatin being transformed after $20 \mathrm{~h}$ of hydrolysis. After liquid liquid extraction, resveratrol purity was $82 \%$ in the organic phase, and increased to $92 \%$ after TONSIL bleaching and insolubilisation in water.

Aknowledgments. This study was supported by SC Salmed Farma SRL: contract no. 127/23.09.2016, ID /COd My SMIS: P_39_307/104965

\section{References}

1.BARBA, F.J., ZHU, Z. , KOUBAA, M., SANT'ANA, A.S., ORLIEN, V., Trends Food Sci.Technol., 49, 2016 , p. 96.

2TOCIU, M., TODASCA M.C., MIHALACHE, M., ARTEM, V., HANGANU, A., Rev. Chim (Bucharest), 69, no.1, 2018, p. 130.

3.TOURNOUR, H.H., SEGUNDOC, M.A., MAGALHÃES, L.M., BARREIROS, L., QUEIROZ J., CUNHA, L.M, Ind. Crops Prod., 74, 2015, p. 397. 4AMRI, A., CHAUMEIL, J.C., SFAR, S., CHARRUEAU, C., J. Control. Release, 158, 2012, p. 182

5.CHENG, V. J., EL-DIN, A., BEKHIT, A., MCCONNELL M., MROS, S., ZHAO, J., Food Chemistry, 134, 2012 , p. 474.

6.BUCIĆ-KOJIĆ, A., PLANINIĆ, M., TOMAS, S., BILIĆ, M., VELIĆ, D., J. Food Eng., 81, 2007, p. 236.

7.WANG, D.G., LIU, W.Y., CHEN, G.T., J. Pharm. Anal., 3, No. 4, 2013, p. 241.

8.RATNASOORIYA, C. C., VASANTHA RUPASINGHE, H. P., FOOD CHEM. 134, 2012, p. 625

9.CHO, Y.-J., HONG, J.-Y., CHUN, H. S., LEE, S. K., MIN, H.-Y., J. Food Eng., 77, 2006, p. 725.

10.ALEXANDRU, L., BINELlO, A., MANTEGNA, S., BOFFA L., CHEMAT,F., CRAVOTTO, G., C. R. Chimie, 17, 2014, p. 212.

11. VATAI, T., ŠKERGET, M., KNEZ, Ž., J. Food Eng., 90, 2009, p. 246.

12.JIN, S., YANG, B., CHENG, Y., TAN, J., KUANG, H., FU, Y., BAI, X., XIE, H., GAO,Y., EFFERT, LV., C. T., Food Bioprod Process, 102, 2017, p. 177.

13.CAI, Y., Studies on extracting of resveratrol and its biological activities, Bejing. Master degree thesis of Beijing University of Chemical Technology, 2010.

14.YANG, J., Food Nutr. China, 1, 2009, p. 17-19.

15.MA, D., Studies on the Extraction and Separation technology of Emodin and Resveratrol from Polygonum Cuspidatum. Tianjin. Master degree thesis of Tianjin University, 2006.

16.CAO, Y., Studies on the extraction and purification of resveratrol from Polygonum Cuspidatum, Changsha. Master degree thesis of Hunan Agricultural University, 2001

Manuscript received: 20.03.2019 